\title{
Editorial: Redefining Length
}

A goal for Letters and short papers is to efficiently and concisely communicate key results and findings. Authors work hard in many cases to maximize the material presented within the constraints of the printed page. Since page formatting occurs after scientific review, and despite conscientious attempts by many authors to adhere to APS style when submitting their work, formatting changes are often required; our current scheme to estimate length, in spite of our best efforts, is fairly inaccurate. This naturally leads to frustration, especially when authors are faced with the arduous task of further editing and revising their work during the proof stage, which sometimes leads to concessions in the clarity of the text and the quality and quantity of referencing. The tension between clearly communicating the primary research results of an investigation and giving proper attribution to prior literature frequently leads to compromises that can be detrimental to physics research.

Technological changes have moved publishing to electronic-first publication where the print version has been relegated to simply another display mode. Distribution in HTML and EPUB formats, for example, changes the reading environment and reduces the need for strict pagination. Therefore, in an effort to streamline the calculation of length, the APS journals will no longer use the printed page as the determining factor for length. Instead the journals will now use word counts (or word equivalents for tables, figures, and equations) to establish length; for details please see http://publish.aps.org/authors/length-guide. The title, byline, abstract, acknowledgment, and references will not be included in these counts allowing authors the freedom to appropriately credit coworkers, funding sources, and the previous literature, bringing all relevant references to the attention of readers. This new method for determining length will be easier for authors to calculate in advance, and lead to fewer length-associated revisions in proof, yet still retain the quality of concise communication that is a virtue of short papers.

We will also use this opportunity to integrate references contained in Supplemental Material into the paper. This will expand the ease by which readers can gain a broad perspective over the relevant literature and help to insure that these articles receive the credit they deserve.

This new method for constraining length will be implemented immediately. You may spot the effect of this change mainly in PRL where we fully expect to see a good number of Letters run onto a fifth page. We hope that authors will find this method easier to use, reduce delays in the review and proof stages, and lead to more comprehensive referencing.

Gene D. Sprouse

Editor in Chief

Published 11 July 2011

American Physical Society

DOI: 10.1103/PhysRevSTPER.7.020001

PACS numbers: 01.30.Ww

Published by the American Physical Society under the terms of the Creative Commons Attribution 3.0 License. Further distribution of this work must maintain attribution to the author(s) and the published article's title, journal citation, and DOI. 